\title{
Preformulation and preliminary formulation studies of mesalazine gastro-resistant tablets
}

\author{
Adriana Aurelia CHIS ${ }^{1,2}$, Isabela CRACIUN ${ }^{1,2}$, Carmen Maximiliana DOBREA ${ }^{1,2}$, \\ Felicia Gabriela GLIGOR ${ }^{1}$, Angela BARBAT ${ }^{2}$, Luca Liviu RUS ${ }^{1,2}$ \\ ${ }^{1}$ Preclinic Department, Faculty of Medicine, Lucian Blaga University, Sibiu, Romania \\ ${ }^{2}$ Polisano Pharmaceuticals SA, Sibiu, Romania
}

\begin{abstract}
Mesalamine, 5-aminosalicylic acid or mesalazine is the standard therapy of inflammatory bowel disease. A small number of pharmaceutical dosage forms with mesalazine are on the market.

The aim of this study was preformulation and preliminary formulation studies of oral gastro-resistant tablets containing $500 \mathrm{mg}$ mesalazine. The reasons why a gastro-resistant tablet was chosen are: increased compliance of the patient, increased chemical stability and modified release modulation (mesalazine has a local effect on mucosa). The raw materials were of pharmaceutical grade. The following analytical techniques were involved: differential scanning chromatography (DCS), in vitro release, particle size determination, high performance liquid chromatography (HPLC). The compatibility of mesalazine with several excipients was tested using DSC. Wet granulation of mesalazine and starch showed that the fourth (LM04) formulation generates the highest amount (69.1\%) of granules in the range of 1000-300 $\mu \mathrm{m}$. Oblong tablets (pilot batches) were produced. The cores were coated with an enteric coating acrylic agent in order to achieve gastro-resistance. A new gastro-resistant tablets mesalazine formulation was developed by means of wet granulation, tableting (oblong tablets) and coating.
\end{abstract}

Keywords: mesalazine, gastro-resistant tablets, pharmaceutical development, wet granulation, gastro-resistant coating

\section{INTRODUCTION}

Mesalamine, 5-aminosalicylic acid or mesalazine is the standard therapy of inflammatory bowel disease.

Ulcerative colitis and Crohn's disease are the main manifestation forms in this category [1]. Recent studies show a growth of the prevalence of the disease in recent years [2]. A small number of oral and rectal pharmaceutical forms containing MSZ are available, individual doses between $250 \mathrm{mg}$ and 2,000 $\mathrm{mg}[3,4]$. Several research directions target the direct delivery of this substance to the intestine: formulation of multiparticulate systems (pellets coated with a polymer resulting in a $\mathrm{pH}$ dependent release system), formulation of composite materials (especially cross-linked chitosan with glutaraldehyde) [1,5-7].

The aim of this study was preformulation and preliminary formulation studies of oral gastro-resistant tablets containing $500 \mathrm{mg}$ mesalazine. This pharmaceutical form was selected due to a couple of reasons. Oral pharmaceutical forms increase the compliance to the prescribed treatment [8] and gastro-resistant coating ensures both the protection against environmental destabilizing factors such as 
light and oxygen and the controlled release of the active pharmaceutical ingredient [9].

\section{MATERIAL AND METHODS}

\section{Materials}

MSZ (Erregiere), microcrystalline cellulose (JRS Pharma), partially pregelatinized maize starch (Colorcon), povidone (BASF), magnesium stearate (Mosselman), lactose monohydrate (Meggle), croscarmellose sodium (FMC BioPolymer), colloidal silicon dioxide (Evonik), talc (Luzenac), calcium carbonate (Shangai Nuoceng Pharmaceuticals). All the components were of pharmaceutical grade and complied with the relevant monographs of the European Pharmacopoeia, 9th edition [10]. Coating system: Opadry clear (Colorcon), Triethylcitrate (Colorcon), Acryl-Eze (Colorcon). Also purified water (Ph. Eur., 2016) was used in the process.

Process steps and equipment - laboratory batches weighing - balance (Mettler Toledo), wet granulation - fluid bed granulator (Bosch), manual blending, tableting - eccentric press (Riva).

Process steps and equipment - pilot batches weighing - balances and scales (Mettler Toledo), wet granulation - fluid bed granulator (Bosch), blending blender (Servolift), tableting - rotary tableting press (Fette 102i), oblong punch 18.2/7.7 mm, coating coating equipment ( $\mathrm{O}^{\prime} \mathrm{Hara}$ Technologies).

\section{Methods}

Differential scanning calorimetry (DSC): Netzsch calorimeter, model DSC 200F3 Maia. Oven DSC 200F3; aluminum sample pans, $40 \mathrm{~mL} / \mathrm{min}$ nitrogen, probe weight $7 \mathrm{mg}$, start temperature $30^{\circ} \mathrm{C}$, end test temperature $400 \circ \mathrm{C}$. The test was conducted for the active substance and the excipients, followed by the combinations active ingredient-excipient in a ratio of 1: 1.

Granulometry analytical sieve shaker Retsch AS 200, test time 4 minutes, $1 \mathrm{~mm} / \mathrm{g}$ amplitude, $10 \mathrm{~s}$ interval, sieves $1 \mathrm{~mm}, 720 \mu \mathrm{m}, 300 \mu \mathrm{m}, 100 \mu \mathrm{m}$ and $50 \mu \mathrm{m}$; $100 \mathrm{~g}$ mesalazine probe.

In process-control height, diameter and hardness tester - Erweka TBH 225 TD, friability tester - Erweka TDR 100, disintegration tester - Erweka TZ 72. The tests were conducted in accordance to compendial references [10].
Dissolution tests Agilent technologies 708-DS Dissolution Apparatus (rotating paddle). Dissolution media: $0.1 \mathrm{~N}$ clorhidric acid, residence time 120 minutes, followed by disodium hydrogen phosphate buffer $\mathrm{pH} 6.8$ residence time 15, 30, 45, 60, 120 minutes.

Identification and assay of MSZ and impurities High Performance Liquid Cromatography (HPLC): Agilent Technologies 1260 Infinity. The tests were conducted in accordance to compendial references $[10,11]$.

For tablets assay, a Xterra MS C18 chromatographic column was used $(25 \mathrm{~cm} \times 4.6 \mathrm{~mm}$, octadecylsilyl silica gel $-5 \mu \mathrm{m})$, mobile phase consisted of a $0.69 \%(\mathrm{~m} / \mathrm{w})$ solution of $\mathrm{KH} 2 \mathrm{PO} 4$, adjusted to $\mathrm{pH}$ 6.2. Elusion was isocratic, flow rate $1 \mathrm{~mL} / \mathrm{min}$, temperature of the column was $40^{\circ} \mathrm{C}$, detection UV at $240 \mathrm{~nm}$.

\section{RESULTS AND DISCUSSION}

In order to obtain gastro-resistant tablets containing $500 \mathrm{mg}$ MSZ, several excipients were selected. The compatibility of the API with these excipients was tested using DSC (Figure 1).

A mixture ratio of 1:1 MSZ and each of the following: croscarmellose sodium, magnesium stearate, povidone showed a different profile compared to the individual behavior, however any of the mentioned excipients are employed in small quantities in oral solid dose formulations, the actual API:excipient ratio in this case being 1:0.05 $(\mathrm{w} / \mathrm{w})$, reducing the potential negative influence of the excipient. No interactions were revealed between mesalazine and Aerosil, starch, talc and lactose.

A formulation containing MSZ, starch, croscarmellose sodium, magnesium stearate was agreed.

MSZ has poor flow capacity, rendering inefficient the process of direct compacting. The granulation of the API offers the possibility to significantly modify this inconvenient. It also prevents the components of the powder mixture from being separated; it improves the compression properties of the mixture by reducing the proportion of air between the particles and reduces the compressive force needed for tableting [12].

Four laboratory batches were developed LM01, LM02, LM03 and LM04. For the first laboratory batch wet granulation was performed using MSZ and starch, the ratio was $10: 0.5$. For the second laboratory batch granulation was performed increasing the binder 

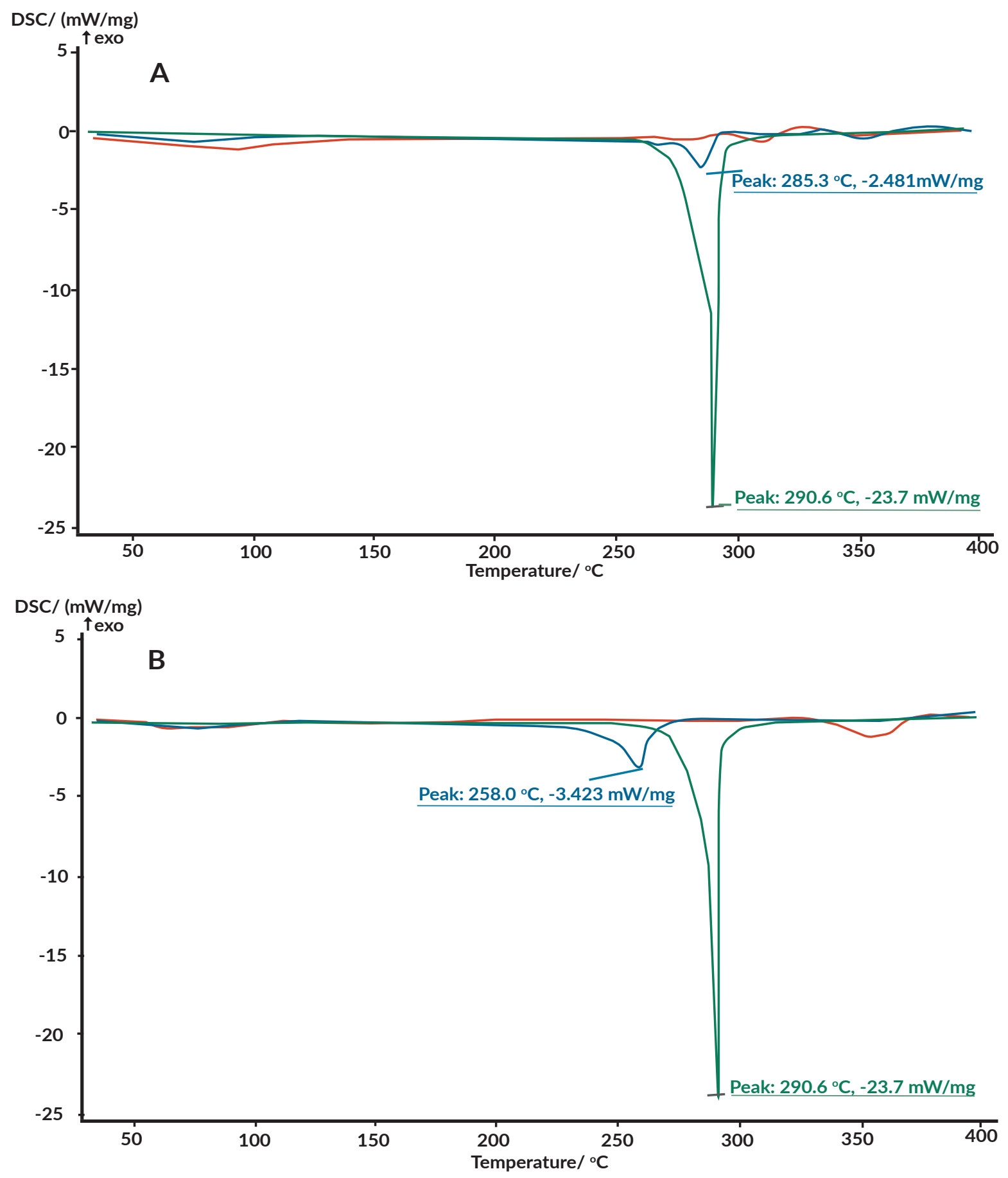

FIGURE 1. DSC analysis of mesalazine and excipients ( $A$ - Starch; B - Magnesium stearate; Mesalazine - green; Excipient - red and Mesalazine:Excipient 1:1 - blue)

TABLE 1. The composition of granule - laboratory batches

\begin{tabular}{|l|c|c|c|c|}
\hline & \multicolumn{4}{|c|}{ Quality (mg/tablet) } \\
\hline Raw material & LM01 & LM02 & LM03 & LM04 \\
\hline Mesalazine & 500 & 500 & 500 & 500 \\
\hline Starch & 25.00 & 37.5 & 50 & 66 \\
\hline Purified water* & 475 & 462.5 & 450 & 484 \\
\hline Granule (total) & 525 & 537,5 & 550 & 566 \\
\hline
\end{tabular}

quantity, API: binder ratio 10:1. The third batch employed the ratio 10:0.75 and the last one 10:1.2 (Table 1). A granulometric comparison of the batches (Figure 2) shows that the fourth formulation generates the highest amount $(69.1 \%)$ of granules in the range of $1000-300 \mu \mathrm{m}$.

*Not found in finished intermediate product (granule) 


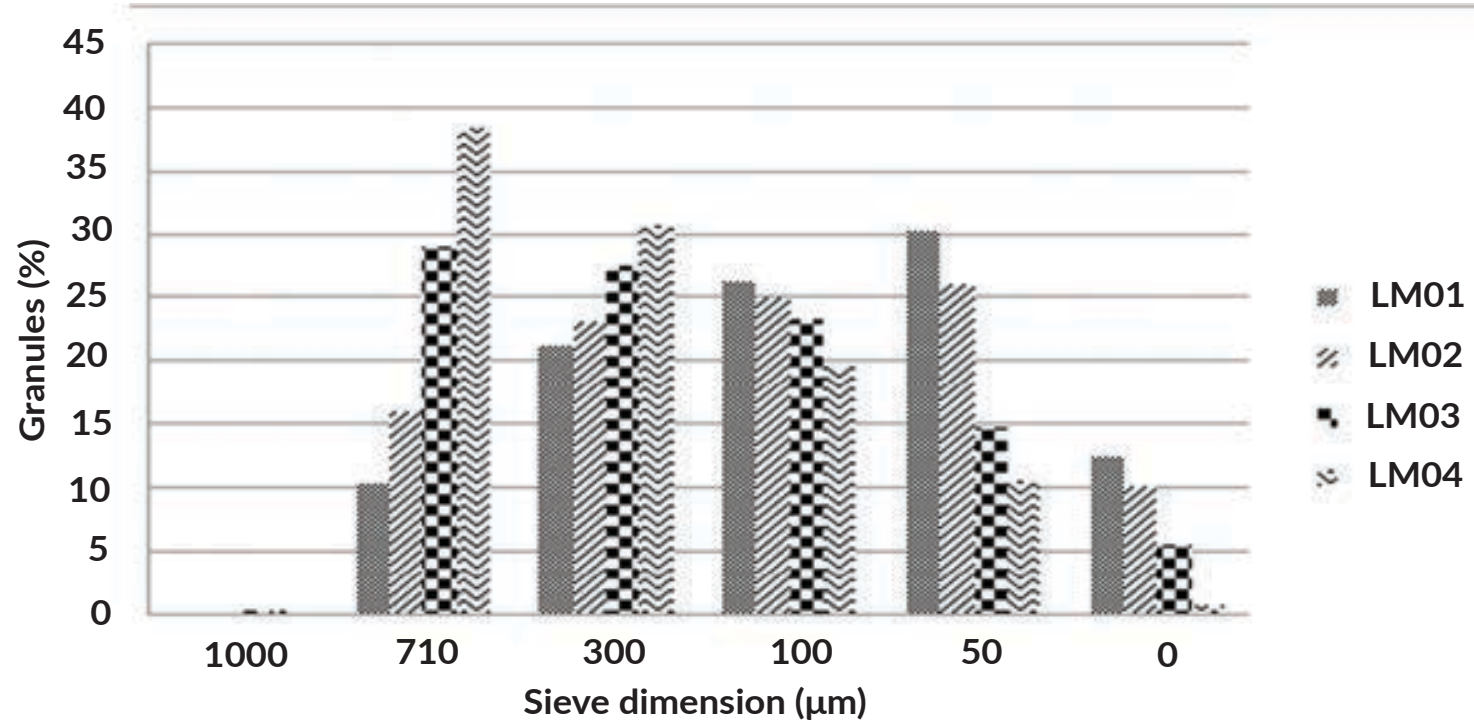

FIGURE 2. The granulometric distribution of mesalazine granules - laboratory batches

Each of the granule batches were mixed with similar amounts of cellulose, and identical amounts of croscarmellose sodium, colloidal silica, talc, and magnesium stearate, in order to obtain $800.00 \mathrm{mg}$ cores. Batches LM01 to LM03 had low apparent powder density and uneven average mass. Batch LMO4 showed steadier characteristics. However, the tablet hardness was low, an inconvenient for the coating process. The augmentation of the compression force did not improve the results. A change in the formulation was needed in order to optimize the tableting process.

Laboratory batch LM05 was developed. The granulation formula of LMO4 was employed and the diluent was modified. Small amounts of calcium carbonate significantly improve powder compressibility [13]. Calcium carbonate was added to the mixture, representing $4 \%$ of the core weight, and the cellulose amount was diminished accordingly. This formula had better results than the previous. Due to the trend of the results, a new laboratory formulation was developed, LM06, increasing the calcium carbonate concentration to $8 \%$ of the core weight, and the cellulose amount was diminished accordingly.

A scale-up of the sixth laboratory formulation led to the first pilot batch PM01. The granulation of the pilot batch had similar results to the laboratory batch, rendering $64.5 \%$ granules in the range of $1000-300$ $\mu \mathrm{m}$.

The tableting process was carried at high speed, and the resulting cores had good pharmacotechnical properties. Also, a quantitative analysis of MSZ was carried out by HPLC (Figure 3).

In order to test the robustness of the granulation and tableting processes, other two pilot batches were produced. The process was reproducible (Table 2 ).

DAD1 $\mathrm{A}$ Sig $=240,4$ Ref $=360,100$ MESALAZINA

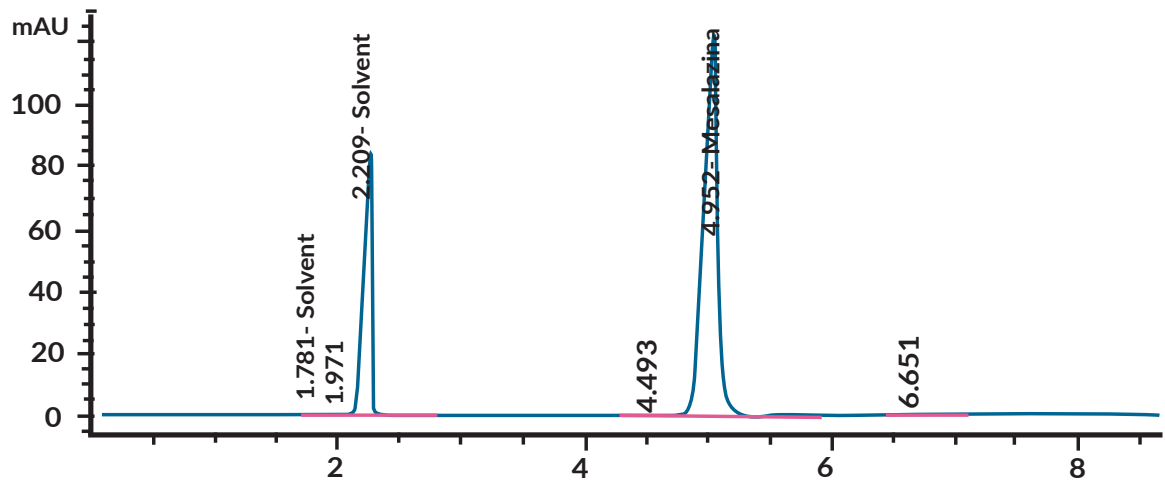

FIGURE 3. Mesalazine chromatogram 
The controlled release of MSZ in the intestines can be achieved using gastro-resistant coating [14]. We employed an aqueous enteric acrylic system: Acryl-eze, weight gain PM01 8\%, PM02 10\%, PM03 $12 \%$. This system was successfully used combined with a

hydroxypropyl methylcellulose base coat [15]. For all three pilot batches an Opadry base coat was used, weight gain $2.2 \%$.

The first two batches failed to pass the gastro-resistance test. The results of the third pilot batch complied with all the requirements of this phase.

The dissolution profile of the new developed MSZ generic was compared to that of Salofalk ${ }^{\circledR} 500$ $\mathrm{mg}$ gastro-resistant tablets, in order to preliminary asses therapeutic equivalence [16]. Experimental results of dissolution tests of $\mathrm{PMO3}$ MSZ 500 mg gastro-resistant tablets showed a $9.7 \%$ similarity factor to the original pharmaceutical product (Figure 4).

The gradual degradation of the coating film of all the tablets, with progressive release of the active substance, is observed approximately 5 minutes after the initiation of the dissolution process. The first sampling operation is performed at 15 minutes, at which point the film of all tablets is already completely dissolved. For the generic product of batch PMO3 the active substance is released in a considerable proportion (78.00\%), unlike the original product releasing a small amount of mesalazine (2.40\%). The amount of active substance continues to increase for both products. The generic product releases the active substance almost completely
TABLE 2. Pharmacotechnical characteristics of mesalazine cores - pilot batches

\begin{tabular}{|c|c|c|c|c|}
\hline & & \multicolumn{3}{|c|}{ Batch } \\
\hline Characteristic & Admissibility crietria & PM01 & PM02 & PM03 \\
\hline Average mass (mg) & $\begin{array}{c}800 \pm 5 \%[760.0- \\
840.0] \\
\end{array}$ & 798.2 & 790.6 & 792.4 \\
\hline Hardness $(\mathrm{N})$ & $200-350$ & 245 & 225 & 271 \\
\hline Thickness (mm) & $6.3-6.5$ & 6.3 & 6.4 & 6.3 \\
\hline Friability (\%) & No more than $1 \%$ & 0.16 & 0.15 & 0.15 \\
\hline Water (\%) & Maximum 4\% & 1.66 & 1.70 & 1.68 \\
\hline \multirow[b]{2}{*}{ Identification } & $\begin{array}{l}\text { IR spectrum } \\
\text { according to the } \\
\text { reference }\end{array}$ & Complies & Complies & Complies \\
\hline & $\begin{array}{l}\text { Retention time of } \\
\text { the test solution } \\
\text { similar to the } \\
\text { standard solution }\end{array}$ & Complies & Complies & Complies \\
\hline Assay (mg/tablet) & $\begin{array}{c}500 \pm 5 \%[475.0- \\
525.0]\end{array}$ & 505.7 & 492.3 & 492.8 \\
\hline \multicolumn{5}{|c|}{ Chemical related impurities (\%) } \\
\hline Impurity H & $\leq 0.3$ & ND* & ND* & ND* \\
\hline Impurity F & $\leq 0.1$ & ND* & ND* & ND* \\
\hline Impurity J & $\leq 0.1$ & ND* & ND* & ND* \\
\hline Impurity O & $\leq 0.1$ & ND* & ND* & ND* \\
\hline Impurity P & $\leq 0.1$ & ND* & ND* & ND* \\
\hline Impurity E & $\leq 0.05$ & ND* & ND* & ND* \\
\hline Impurity G & $\leq 0.05$ & ND* & ND* & ND* \\
\hline Impurity L & $\leq 0.05$ & ND* & ND* & ND* \\
\hline Impurity M & $\leq 0.05$ & ND* & ND* & ND* \\
\hline Impurity R & $\leq 0.05$ & ND* & ND* & ND* \\
\hline Other impurities & $\leq 0.05$ & ND* & ND* & ND* \\
\hline Total impurities & $\leq 0.5$ & ND* & ND* & ND* \\
\hline \multicolumn{5}{|c|}{ Impurity $A$ and $C$ (ppm) } \\
\hline A: 4-aminophenol & $\leq 200$ & ND* & ND* & ND* \\
\hline C: 2-aminophenol & $\leq 200$ & 25.7 & 24.9 & 25.5 \\
\hline \multicolumn{5}{|l|}{ Impurity K (ppm) } \\
\hline Aniline & $\leq 10$ & ND* & ND* & ND* \\
\hline
\end{tabular}

*ND $=$ Not detected

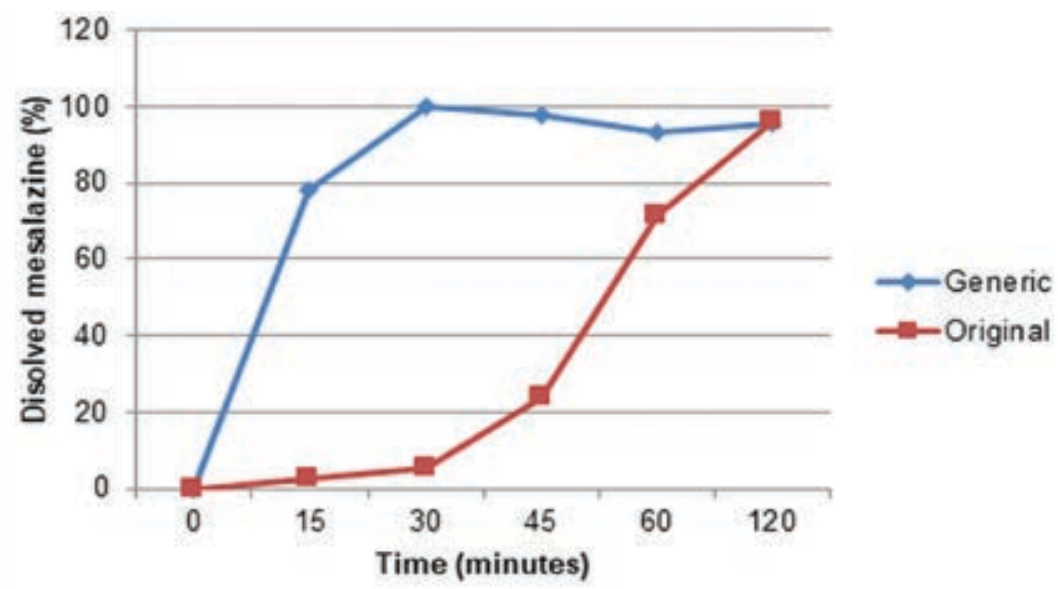

FIGURE 4. The dissolution profile of the generic product PM03 versus the original product 
(99.70\%) after 30 minutes of dissolution, unlike the original product which reaches the maximum MSZ concentration after 120 minutes. This immediate release of the API would suggest that the generic product could start to produce a therapeutic effect in a shorter amount of time compared to the original. After 15 minutes there is almost complete dissolution of the active substance in the generic product tablets.

A first possible solution to reduce the speed of dissolution would be to decrease the super disintegrant quantity. For the formulation of pilot batch PM04 we applied a 50\% reduction of croscarmellose sodium percentage compared to PM03, specifically $1.5 \%$ instead of $3 \%$. Cellulose was used for weight correction. The similarity factor increased to $25.8 \%$ for MSZ gastro-resistant tablets batch PM04 (Figure 5).

Observing the allure of the curves of PM04, PM03 and the original product (Figure 4 and 5 ), a further development of this study would suggest testing superdisintegrant ratios in between the values of the two generics. Other possibilities to expand the study include variations of the granulation process [17] and variations of excipient ratio and type [18].

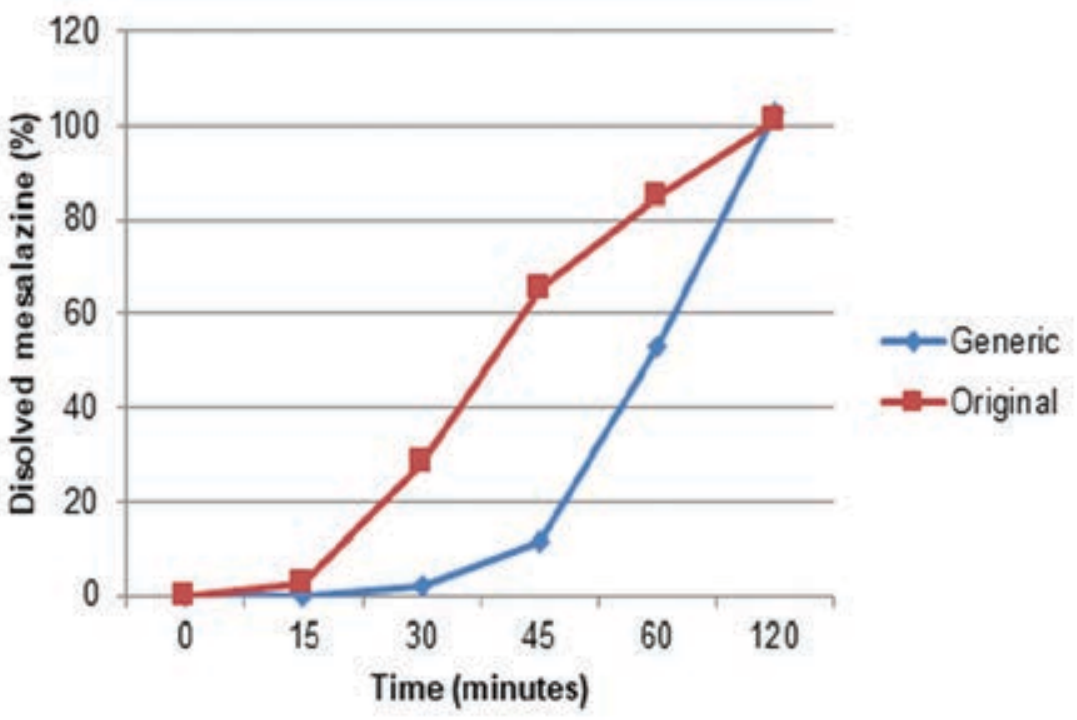

FIGURE 5. The dissolution profile of the generic product PM04 versus the original product

\section{CONCLUSIONS}

Mesalazine is the standard therapy of inflammatory bowel disease. Several research directions target the direct delivery of this substance to the intestine. A new formulation of MSZ 500 mg gastro-resistant tablets was developed. A new gastro-resistant tablets mesalazine formulation was developed by means of wet granulation, tableting (oblong tablets) and coating. Each step of the process was controlled, and the intermediary product was analyzed. Further studies to modulate the dissolution profile of the tablets are in progress.

Conflict of interest: none declared Financial support: none declared

\section{REFERENCES}

1. Kandula M, Sunil Kumar KB, Palanichamy S, Rampal A. Discovery and preclinical development of a novel prodrug conjugate of mesalamine with eicosapentaenoic acid and caprylic acid for the treatment of inflammatory bowel diseases. Int Immunopharmacol. 2016;40: 443-51.

2. Dahlhamer JM, Zammitti EP, Ward BW, Wheaton AG, Croft JB. Prevalence of inflammatory bowel disease among adults aged $\geq 18$ years - United States. MMWR Morb Mortal Wkly Rep. 2016;65(42):1166-9.

3. Ye B, van Langenberg DR. Mesalazine preparations for the treatment of ulcerative colitis: Are all created equal? World J Gastrointest Pharmacol Ther. 2015;6:138-40.

4. Perrotta C, Pellegrino P, Moroni E. Five-Aminosalicylic Acid: An Update for the Reappraisal of an Old Drug. World J Gastrointest Pharmacol Ther. 2015;6(4):137-44.

5. Santana ACSGV, Sobrinho JLS, Silva Filho ECD, Nunes LCC. Obtaining the palygorskite: chitosan composite for modified release of 5-aminosalicylic acid. Mater Sci Eng C Mater Biol Appl. 2017:73:245-51.
6. Neufeld L, Bianco-Peled H. Designing a biocompatible hydrogel for the delivery of mesalamine. Int J Pharm. 2015;491(1-2):170-9.

7. Déo SC, Andreazza IF, Possamai JC. Development of mesalazine pellets coated with methacrylic-derived polymer. Braz J Pharm Sci. 2011;47(1):103-109.

8. Shahiwala A. Formulation approaches in enhancement of patient compliance to oral drug therapy. Expert Opin Drug Deliv. 2011;8(11):1521-9.

9. Siepmann J, Siepmann F. Stability of aqueous polymeric controlled release film coatings. Int J Pharm. 2013;457(2):437-45.

10. Council of Europe. European Pharmacopoeia. 9th ed., Strasbourg: Council of Europe; 2016.

11. British Pharmacopoeia Commission. British Pharmacopoeia, London: TSO; 2016.

12. Shanmugam S. Granulation techniques and technologies: recent progresses. Bioimpacts. 2015; 5(1):55-63.

13. Stirnimann T, Atria S, Schoelkopf J, Gane PA, Alles R, Huwyler J et al. Compaction of Functionalized Calcium Carbonate: A Porous and Crystalline Microparticulate Material with a Lamellar Surface. Int J Pharm. 2014;466(1-2):266-75. 
14. Lamoudi L, Chaumeil JC, Daoud K. Development of gastro intestinal sustained release tablet formulation containing acryl-EZE and pH-dependent swelling HPMC K 15. M Drug Dev Ind Pharm. 2012;38(5):515-20.

15. Hashmat D, Shoaib MH, Mehmood ZA, Bushra R, Yousuf RI, Lakhani F. Development of enteric coated flurbiprofen tablets using Opadry/Acryl-Eze system-a technical note. AAPS PharmSciTech. 2008;9(1):116-21.

16. Klein S. Similar in vitro drug release as a surrogate of therapeutic equivalence of locally acting gastrointestinal products--what is the right in vitro method? Pharmazie. 2015;70(8):535-42.
17. Fayed MH, Abdel-Rahman SI, Alanazi FK, Ahmed MO, Tawfeek HM, Ali BE. High shear granulation process: assessing impact of formulation variables on granules and tablets characteristics of high drug loading formulation using design of experiment methodology. Acta Pol Pharm. 2017;74(2):551-64.

18. Goyanes A, Hatton GB, Merchant HA, Basit AW. Gastrointestinal release behaviour of modified-release drug products: dynamic dissolution testing of mesalazine formulations. Int J Pharm. 2015;484(1-2):103-8. 